\title{
Left-orderable fundamental groups and Dehn surgery on genus one 2-bridge knots
}

\author{
RYOTO HAKAMATA \\ MASAKAZU TERAGAITO
}

\begin{abstract}
For any hyperbolic genus-one 2-bridge knot in the 3-sphere, such as any hyperbolic twist knot, we show that the manifold resulting from $r$-surgery on the knot has left-orderable fundamental group if the slope $r$ lies in some range, which depends on the knot.
\end{abstract}

$57 \mathrm{M} 25 ; 06 \mathrm{~F} 15$

\section{Introduction}

A non-trivial group $G$ is said to be left-orderable if it admits a strict total ordering that is invariant under left-multiplication. Thus, if $g<h$ then $f g<f h$ for any $f, g, h \in G$. Many groups that arise in topology, such as orientable surface groups, knot groups, braid groups, are known to be left-orderable. In 3-manifold topology, it is natural to ask which 3-manifolds have left-orderable fundamental groups. Toward this direction, there is very recent evidence of connections between Heegaard Floer homology and left-orderability of fundamental groups. More precisely, Boyer, Gordon and Watson [3] conjecture that an irreducible rational homology 3 -sphere is an $L$-space if and only if its fundamental group is not left-orderable. An $L$-space is a rational homology 3-sphere $Y$ whose Heegaard Floer homology group $\widehat{H F}(Y)$ is a free abelian group with rank equal to $\left|H_{1}(Y ; \mathbb{Z})\right|$ (Ozsváth and Szabó [18]). Recently $L$-spaces have become an object of interest, and it is an open problem to characterize $L$-spaces without mentioning Heegaard Floer homology. An affirmative answer to the above conjecture would give an algebraic characterization of $L$-spaces. Boyer, Gordon and Watson confirmed the conjecture for several classes of 3-manifolds, including Seifert fibered manifolds and Sol-manifolds. Also, they showed that if $-4<r<4$ then $r$-surgery on the figure-eight knot yields a 3-manifold whose fundamental group is left-orderable. Later, Clay, Lidman and Watson [6] confirmed the same conclusion for $r= \pm 4$. Since the figure-eight knot cannot yield $L$-spaces by non-trivial Dehn surgery [18], these give supporting evidence of the conjecture. Other results toward this direction appear in Clay and Teragaito [7], Li and Roberts [16] and Teragaito [22]. 
For a knot $K$ in the 3-sphere, we call a slope $r$ left-orderable if the manifold $K(r)$ resulting from $r$-surgery on $K$ has left-orderable fundamental group. In this paper, a slope is sometimes identified with its standard parameter in $\mathbb{Q} \cup\{1 / 0\}$ (see Rolfsen [21]). In particular, the meridional slope corresponds to $1 / 0$. It is known that any hyperbolic 2 -bridge knot does not admit Dehn surgery yielding an $L$-space [18]. Hence any slope but $1 / 0$ is expected to be left-orderable for a hyperbolic 2 -bridge knot, if we believe the above conjecture. However, showing this seems beyond our reach at the moment. The purpose of this paper is to give the ranges consisting of left-orderable slopes for all hyperbolic genus-one 2-bridge knots. These include hyperbolic twist knots.

For non-zero integers $m$ and $n$, let $K(m, n)$ be the 2-bridge knot $S(4 m n+1,2 m)$ in Schubert's normal form as illustrated in Figure 1. Here, the twists in the vertical box are left-handed (resp. right-handed) if $m>0$ (resp. $m<0$ ), but those in the horizontal box are right-handed (resp. left-handed) if $n>0$ (resp. $n<0)$. For example, $K(1,-1)$ is the right-handed trefoil, and $K(1,1)$ is the figure-eight knot. By symmetry, $K(m, n)$ and $K(-n,-m)$ are isotopic. Since $K(-m,-n)$ is the mirror image of $K(m, n)$, we may consider only $m>0$. With the exception of the trefoil, $K(m, n)$ is hyperbolic. It is also well known that any genus-one 2-bridge knot is equivalent to $K(m, n)$ for some $m, n$ (see Burde and Zieschang [5]).
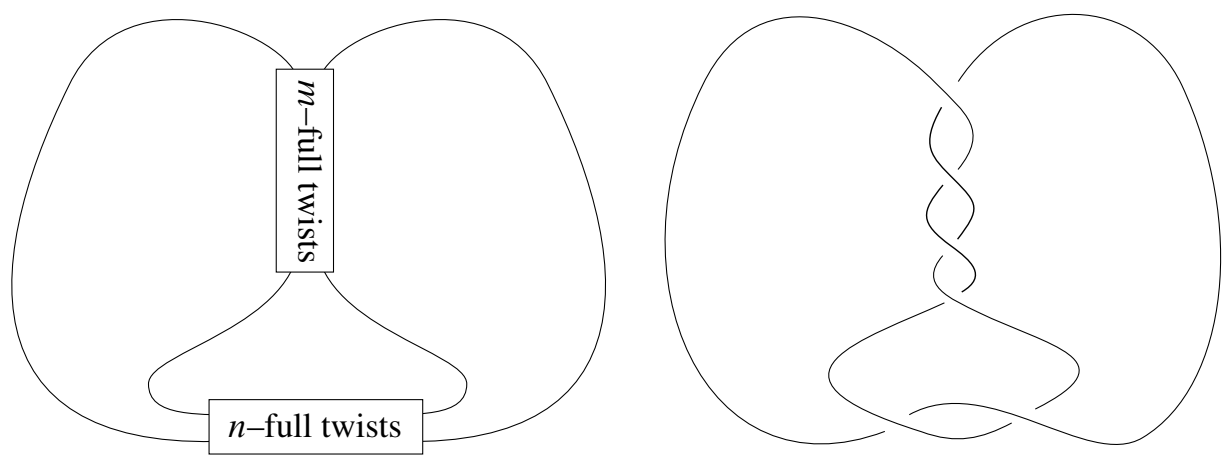

Figure 1: A genus-one 2-bridge knot $K(m, n)$ and $K(2,1)$

Theorem 1.1 Let $K=K(m, n)$ be a hyperbolic genus-one 2-bridge knot $S(4 m n+$ $1,2 m)$ in the 3-sphere as illustrated in Figure 1. Let $I$ be the interval defined by

$$
I= \begin{cases}(-4 n, 4 m) & \text { if } n>0, \\ {[0, \max \{4 m,-4 n\})} & \text { if } m>1 \text { and } n<-1, \\ {[0,4]} & \text { otherwise. }\end{cases}
$$

Then any slope in $I$ is left-orderable. That is, the fundamental group $\pi_{1}(K(r))$ of the resulting manifold $K(r)$ of $r$-surgery is left-orderable if $r \in I$. 
In particular, $K(1, n)$ and $K(m, \pm 1)$ are twist knots. As mentioned before, $K(m,-1)$ is isotopic to $K(1,-m)$, and $K(m, 1)$ is the mirror image of $K(1, m)$.

Corollary 1.2 Let $K(1, n)$ be the $n$-twist knot with $n \neq-1$. If $n>0$, then any slope in the interval $(-4 n, 4]$ is left-orderable. If $n<-1$, then any slope in the interval $[0,4]$ is left-orderable.

A similar difference between positive and negative twist knots is also found in Dąbkowski, Przytycki and Togha [9], and Peters [19].

Remark 1.3 As mentioned before, the trefoil is the only non-hyperbolic knot among genus-one 2-bridge knots. For the (right-handed) trefoil, it is known that $r$-surgery yields an $L$-space if and only if $r \geq 1$. Since the resulting manifold under Dehn surgery is either a Seifert fibered manifold or a connected sum of two lens spaces, this is also equivalent to $\pi_{1}(K(r))$ being not left-orderable. See Boyer, Gordon and Watson [3], and Clay and Watson [8].

Our argument works even for the figure-eight knot $K(1,1)$, and it is much simpler than that in [3], which involves character varieties.

Finally, Tran [23] informed us that he obtained a result similar to Theorem 1.1.

\section{Acknowledgments}

We would like to thank the referee for valuable comments, and Anh T Tran for informing us of his result. The second author was partially supported by Japan Society for the Promotion of Science, Grant-in-Aid for Scientific Research (C) 22540088.

\section{Knot groups and two sequences of polynomials}

Let $K=K(m, n)$ and let $G=\pi_{1}\left(S^{3}-K\right)$ be its knot group. We always assume that $m>0$ and $n \neq 0$, unless specified otherwise.

Proposition 2.1 The knot group $G$ admits a presentation

$$
G=\left\langle x, y \mid w^{n} x=y w^{n}\right\rangle,
$$

where $x$ and $y$ are meridians and $w=\left(x y^{-1}\right)^{m}\left(x^{-1} y\right)^{m}$. Furthermore, the longitude $\mathcal{L}$ is given as $\mathcal{L}=w_{*}^{n} w^{n}$, where $w_{*}=\left(y x^{-1}\right)^{m}\left(y^{-1} x\right)^{m}$ is obtained from $w$ by reversing the order of letters. 
This is slightly different from that in Hoste and Shanahan [14, Proposition 1], but both are isomorphic.

Proof We use a surgery diagram of $K$ as illustrated in Figure 2, where $1 / m$-surgery and $-1 / n$-surgery are performed along the second and third components, respectively. Let $\mu_{i}$ and $\lambda_{i}$ be the meridian and longitude of the $i^{\text {th }}$ component.

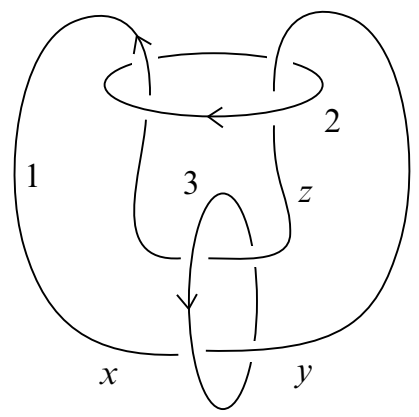

Figure 2: A surgery diagram of $K$

First, $y=\mu_{3}^{-1} x \mu_{3}, z=\mu_{2}^{-1} y \mu_{2}, \lambda_{2}=x^{-1} y$ and $\lambda_{3}=y z^{-1} .1 / m$-surgery on the second component yields a relation $\lambda_{2}^{m} \mu_{2}=1$, so $\mu_{2}=\lambda_{2}^{-m}$. Similarly, $-1 / n-$ surgery on the third component gives $\mu_{3}=\lambda_{3}^{n}$. We remark that the knot group $G$ is generated by $x$ and $y$. This fact can be seen by eliminating $z, \mu_{2}$ and $\mu_{3}$.

Thus

$\lambda_{3}=y \mu_{2}^{-1} y^{-1} \mu_{2}=y \lambda_{2}^{m} y^{-1} \lambda_{2}^{-m}=y\left(x^{-1} y\right)^{m} y^{-1}\left(y^{-1} x\right)^{m}=\left(y x^{-1}\right)^{m}\left(y^{-1} x\right)^{m}$.

Set

$$
w_{*}=\lambda_{3}=\left(y x^{-1}\right)^{m}\left(y^{-1} x\right)^{m} .
$$

Then the relations $y=\mu_{3}^{-1} x \mu_{3}$ and $\mu_{3}=\lambda_{3}^{n}$ give $y \lambda_{3}^{-n}=\lambda_{3}^{-n} x$. This gives

$$
y\left[\left(x^{-1} y\right)^{m}\left(x y^{-1}\right)^{m}\right]^{n}=\left[\left(x^{-1} y\right)^{m}\left(x y^{-1}\right)^{m}\right]^{n} x .
$$

Set $w=\left(x y^{-1}\right)^{m}\left(x^{-1} y\right)^{m}$. Since $\left[\left(x^{-1} y\right)^{m}\left(x y^{-1}\right)^{m}\right]^{n}=\left(x^{-1} y\right)^{m} w^{n}\left(x^{-1} y\right)^{-m}$, (2) changes to $y\left(x^{-1} y\right)^{m} w^{n}\left(x^{-1} y\right)^{-m}=\left(x^{-1} y\right)^{m} w^{n}\left(x^{-1} y\right)^{-m} x$. Вy $y\left(x^{-1} y\right)^{m}=$ $\left(y x^{-1}\right)^{m} y$, we have $\left(y x^{-1}\right)^{m} y w^{n}\left(x^{-1} y\right)^{-m}=\left(x^{-1} y\right)^{m} w^{n}\left(x^{-1} y\right)^{-m} x$. Thus,

$$
\begin{aligned}
y w^{n} & =\left(x y^{-1}\right)^{m}\left(x^{-1} y\right)^{m} w^{n}\left(x^{-1} y\right)^{-m} x\left(x^{-1} y\right)^{m}=w^{n+1}\left(x^{-1} y\right)^{-m} x\left(x^{-1} y\right)^{m} \\
& =w^{n}\left(x y^{-1}\right)^{m}\left(x^{-1} y\right)^{m}\left(x^{-1} y\right)^{-m} x\left(x^{-1} y\right)^{m}=w^{n}\left(x y^{-1}\right)^{m} x\left(x^{-1} y\right)^{m} \\
& =w^{n} x .
\end{aligned}
$$


The longitude is $\mathcal{L}=\mu_{3} \mu_{2} \mu_{3}^{-1} \mu_{2}^{-1}=w_{*}^{n} \mu_{2} w_{*}^{-n} \mu_{2}^{-1}$. We have

$$
\begin{aligned}
\mu_{2} w_{*}^{-n} \mu_{2}^{-1} & =\left(x^{-1} y\right)^{-m}\left[\left(y x^{-1}\right)^{m}\left(y^{-1} x\right)^{m}\right]^{-n}\left(x^{-1} y\right)^{m} \\
& =\left(x^{-1} y\right)^{-m}\left[\left(x^{-1} y\right)^{m}\left(x y^{-1}\right)^{m}\right]^{n}\left(x^{-1} y\right)^{m} \\
& =\left[\left(x y^{-1}\right)^{m}\left(x^{-1} y\right)^{m}\right]^{n}=w^{n} .
\end{aligned}
$$

Thus $\mathcal{L}=w_{*}^{n} w^{n}$.

To describe the Riley polynomial of $K$ in Section 3, we prepare two sequences of polynomials with a single variable $s$.

For non-negative integer $m$, let $f_{m} \in \mathbb{Z}[s]$ be defined by the recursion

$$
f_{m+2}-(s+2) f_{m+1}+f_{m}=0
$$

with initial conditions $f_{0}=1$ and $f_{1}=s+1$. Also, let $g_{m} \in \mathbb{Z}[s]$ be defined by the same recursion

$$
g_{m+2}-(s+2) g_{m+1}+g_{m}=0
$$

with slightly different initial conditions $g_{0}=1$ and $g_{1}=s+2$. We remark that $g_{m}$ is equivalent to the Chebyshev polynomial of the second kind. For convenience, set $f_{-1}=1$ and $g_{-1}=0$.

Lemma 2.2 Let $m \geq 0$. The closed formulas for $f_{m}$ and $g_{m}$ are

$$
f_{m}=\sum_{i=0}^{m}\left(\begin{array}{c}
m+i \\
m-i
\end{array}\right) s^{i}, \quad g_{m}=\sum_{i=0}^{m}\left(\begin{array}{c}
m+1+i \\
m-i
\end{array}\right) s^{i} .
$$

In particular,

(1) all the coefficients of $f_{m}$ and $g_{m}$ are positive integers,

(2) the degree of $f_{m}$ and $g_{m}$ is $m$,

(3) $f_{m}$ and $g_{m}$ are monic.

Proof By induction on $m$; apply the recursive formulas.

Lemma 2.3 For $m \geq 0$, the polynomials $f_{m}, g_{m}$ satisfy the following relations.

(1) $f_{m}+g_{m-1}=g_{m}$

(2) $f_{m}+s g_{m}=f_{m+1}$

(3) $f_{m}^{2}=s g_{m} g_{m-1}+1$ 
Proof These are easily proved by induction on $m$. We prove only (3). It holds when $m=0$. Assume $f_{m}^{2}=s g_{m} g_{m-1}+1$. From (1), (2) and the recursive formula for $g_{m}$,

$$
\begin{aligned}
f_{m+1}^{2} & =\left(f_{m}+s g_{m}\right)^{2}=f_{m}^{2}+2 s f_{m} g_{m}+s^{2} g_{m}^{2} \\
& =\left(s g_{m} g_{m-1}+1\right)+2 s f_{m} g_{m}+s^{2} g_{m}^{2} \\
& =\left(s g_{m} g_{m-1}+1\right)+2 s\left(g_{m}-g_{m-1}\right) g_{m}+s^{2} g_{m}^{2} \\
& =\left(s^{2}+2 s\right) g_{m}^{2}-s g_{m} g_{m-1}+1=s\left((s+2) g_{m}-g_{m-1}\right) g_{m}+1 \\
& =s g_{m+1} g_{m}+1 .
\end{aligned}
$$

Lemma 2.4 If a positive real number is substituted to $s$, then $s g_{m-1}<4 f_{m}$.

Proof Since $s g_{m-1}=f_{m}-f_{m-1}$ by Lemma 2.3, the claim immediately follows from $3 f_{m}+f_{m-1}>0$.

\section{Riley polynomials}

We will construct a family of representations of the knot group $G$ into $\mathrm{SL}_{2}(\mathbb{R})$. Let $F$ be a free group generated by $x$ and $y$. Let $s$ and $t$ be positive real numbers. Define a homomorphism $\rho: F \rightarrow \mathrm{SL}_{2}(\mathbb{R})$ by

$$
\rho(x)=\left(\begin{array}{cc}
\sqrt{t} & 1 / \sqrt{t} \\
0 & 1 / \sqrt{t}
\end{array}\right), \quad \rho(y)=\left(\begin{array}{cc}
\sqrt{t} & 0 \\
-s \sqrt{t} & 1 / \sqrt{t}
\end{array}\right) .
$$

Recall that $w=\left(x y^{-1}\right)^{m}\left(x^{-1} y\right)^{m}$. If $\rho\left(w^{n} x\right)=\rho\left(y w^{n}\right)$, then $\rho$ induces a representation of $G$ into $\mathrm{SL}_{2}(\mathbb{R})$. Although this relation yields four equations, Riley [20] shows that the four equations reduce to a single one. Let $W=\rho(w)$ and $z_{i, j}$ be the $(i, j)$-entry of $W^{n}$. Then $\rho$ induces a non-abelian representation of $G$ if $s$ and $t$ are a pair of solutions of Riley's equation $\phi_{K}(s, t)=0$. The Riley polynomial of $K$ is given by $\phi_{K}(s, t)=z_{1,1}+(1-t) z_{1,2}$. (See also Dubois, Huynh and Yamaguchi [10].)

To calculate the Riley polynomial of $K$, we need to diagonalize the matrix $W$.

Assumption 3.1 In this section, we assume that $n \neq-1$ and that positive real numbers $s$ and $t$ are chosen to satisfy the inequality $s+2<t+1 / t<s+2+4 /\left(s g_{m-1}^{2}\right)$.

Proposition 3.2 For $W=\rho(w)$, we have

$$
W=\left(\begin{array}{cc}
f_{m}^{2}-s t g_{m-1}^{2} & f_{m-1} g_{m-1}-\frac{f_{m} g_{m-1}}{t} \\
s f_{m} g_{m-1}-s t f_{m-1} g_{m-1} & f_{m-1}^{2}-\frac{s}{t} g_{m-1}^{2}
\end{array}\right) .
$$


Proof We prove by induction on $m$. (For the knot $K(m, n)$, we assume $m \neq 0$. However, this proposition holds even for $m=0$.)

If $m=0$, then $w=1$, so $W$ is the identity matrix. It is easy to check that the claim holds.

Assume the conclusion for $m$. Note that

$$
\rho\left(x y^{-1}\right)=\left(\begin{array}{cc}
s+1 & 1 \\
s & 1
\end{array}\right), \quad \rho\left(x^{-1} y\right)=\left(\begin{array}{cc}
s+1 & -1 / t \\
-s t & 1
\end{array}\right) .
$$

Calculate the product

$$
\left(\begin{array}{cc}
s+1 & 1 \\
s & 1
\end{array}\right)\left(\begin{array}{cc}
f_{m}^{2}-s t g_{m-1}^{2} & f_{m-1} g_{m-1}-\frac{f_{m} g_{m-1}}{t} \\
s f_{m} g_{m-1}-s t f_{m-1} g_{m-1} & f_{m-1}^{2}-\frac{s}{t} g_{m-1}^{2}
\end{array}\right)\left(\begin{array}{cc}
s+1 & -1 / t \\
-s t & 1
\end{array}\right) .
$$

By using Lemma 2.3, each entry is identified as desired. For example, the $(1,1)$-entry is given by

$$
\left(\begin{array}{ll}
s+1 & 1
\end{array}\right)\left(\begin{array}{cc}
f_{m}^{2}-s t g_{m-1}^{2} & f_{m-1} g_{m-1}-\frac{f_{m} g_{m-1}}{t} \\
s f_{m} g_{m-1}-s t f_{m-1} g_{m-1} & f_{m-1}^{2}-\frac{s}{t} g_{m-1}^{2}
\end{array}\right)\left(\begin{array}{c}
s+1 \\
-s t
\end{array}\right) .
$$

Then the $(1,1)$-entry is

$$
\begin{aligned}
\left((s+1)^{2} f_{m}^{2}+2 s(s+1) f_{m} g_{m-1}+s^{2} g_{m-1}^{2}\right) & \\
-s t\left((s+1)^{2} g_{m-1}^{2}\right. & \left.+2(s+1) f_{m-1} g_{m-1}+f_{m-1}^{2}\right) \\
= & \left((s+1) f_{m}+s g_{m-1}\right)^{2}-s t\left(f_{m-1}+(s+1) g_{m-1}\right)^{2} .
\end{aligned}
$$

This can be changed further:

$$
\begin{aligned}
\left(f_{m}+s\left(f_{m}+g_{m-1}\right)\right)^{2}-s t & \left(\left(f_{m-1}+s g_{m-1}\right)+g_{m-1}\right)^{2} \\
= & \left(f_{m}+s g_{m}\right)^{2}-s t\left(f_{m}+g_{m-1}\right)^{2}=f_{m+1}^{2}-s t g_{m}^{2}
\end{aligned}
$$

To calculate $W^{n}$ for $|n|>1$, we need to diagonalize $W$. Let $\lambda_{ \pm} \in \mathbb{C}$ be the eigenvalues of $W$. Since $\operatorname{det} W=1, \lambda_{+} \lambda_{-}=1$.

Lemma $3.3 \lambda_{+} \neq \lambda_{-}$

Proof If $\lambda_{+}=\lambda_{-}$, then $\lambda_{+}=\lambda_{-}= \pm 1$. Then $\operatorname{tr} W= \pm 2$.

On the other hand, $\operatorname{tr} W=f_{m}^{2}+f_{m-1}^{2}-s(t+1 / t) g_{m-1}^{2}=s(s+2-t-1 / t) g_{m-1}^{2}+2$ by Proposition 3.2 and Lemma 2.3. Since $g_{m-1} \neq 0$ (recall $m \neq 0$ ), $s>0$ and $s+2<t+1 / t$ by Assumption 3.1, we have $\operatorname{tr} W<2$. If $\operatorname{tr} W=-2$, then

$$
s(t+1 / t-s-2) g_{m-1}^{2}=4,
$$


so $t+1 / t=s+2+4 /\left(s g_{m-1}^{2}\right)$. But this is impossible by Assumption 3.1 again. Hence $\lambda_{+} \neq \lambda_{-}$.

Let $w_{i, j}$ be the $(i, j)$-entry of $W$, and let $P=\left(\begin{array}{cc}w_{1,2} & w_{1,2} \\ \lambda_{+}-w_{1,1} & \lambda_{-}-w_{1,1}\end{array}\right)$.

Lemma $3.4 w_{1,2} \neq 0$

Proof Assume $w_{1,2}=0$. By Proposition 3.2, $w_{1,2}=f_{m-1} g_{m-1}-f_{m} g_{m-1} / t$. Hence $f_{m}=t f_{m-1}$. From the recursion (3), $t f_{m-1}-(s+2) f_{m-1}+f_{m-2}=0$. Since $f_{m-1} \neq 0$, we have $t=s+2-f_{m-2} / f_{m-1}$. Thus $t<s+2$, contradicting Assumption 3.1.

The next result will be used in Section 5.

Lemma $3.5 w_{2,1}=-$ st $w_{1,2}$. Hence $w_{2,1} \neq 0$.

Proof This immediately follows from Proposition 3.2 and Lemma 3.4.

By Lemmas 3.3 and 3.4, det $P=-w_{1,2}\left(\lambda_{+}-\lambda_{-}\right) \neq 0$. A direct calculation gives

$$
P^{-1} W P=\left(\begin{array}{cc}
\lambda_{+} & 0 \\
0 & \lambda_{-}
\end{array}\right)
$$

For any integer $k$, set $\tau_{k}=\left(\lambda_{+}^{k}-\lambda_{-}^{k}\right) /\left(\lambda_{+}-\lambda_{-}\right)$. Since $\tau_{k}$ is symmetric in $\lambda_{+}$and $\lambda_{-}$, it can be expressed as a polynomial of $\operatorname{tr} W=\lambda_{+}+\lambda_{-}$. Also, $\tau_{-k}=-\tau_{k}$, and a recursion $\tau_{k+1}-(\operatorname{tr} W) \tau_{k}+\tau_{k-1}=0$ holds for any integer $k$.

Lemma 3.6 For $W^{n}=\rho\left(w^{n}\right)$, we have

$$
W^{n}=\left(\begin{array}{cc}
w_{1,1} \tau_{n}-\tau_{n-1} & w_{1,2} \tau_{n} \\
w_{2,1} \tau_{n} & \tau_{n+1}-w_{1,1} \tau_{n}
\end{array}\right)
$$

Proof When $n=1$, it is easy to see the conclusion. Let $|n|>1$. The conclusion follows from the matrix calculation of

$$
W^{n}=P\left(\begin{array}{cc}
\lambda_{+}^{n} & 0 \\
0 & \lambda_{-}^{n}
\end{array}\right) P^{-1}
$$

We check only the $(2,1)$-entry here. A direct calculation gives

$$
-\frac{\tau_{n}}{w_{1,2}}\left(\lambda_{+}-w_{1,1}\right)\left(\lambda_{-}-w_{1,1}\right)
$$


for the $(2,1)$-entry. By using tr $W=\lambda_{+}+\lambda_{-}=w_{1,1}+w_{2,2}$ and $\operatorname{det} W=w_{1,1} w_{2,2}-$ $w_{1,2} w_{2,1}=1$,

$$
\begin{aligned}
-\frac{\tau_{n}}{w_{1,2}}\left(\lambda_{+}-w_{1,1}\right)\left(\lambda_{-}\right. & \left.-w_{1,1}\right) \\
& =-\frac{\tau_{n}}{w_{1,2}}\left(1-(\operatorname{tr} W) w_{1,1}+w_{1,1}^{2}\right)=-\frac{\tau_{n}}{w_{1,2}}\left(1-w_{1,1} w_{2,2}\right) \\
& =-\frac{\tau_{n}}{w_{1,2}}\left(-w_{1,2} w_{2,1}\right)=w_{2,1} \tau_{n} .
\end{aligned}
$$

Proposition 3.7 The Riley polynomial of $K$ is

$$
\phi_{K}(s, t)=\left(\tau_{n+1}-\tau_{n}\right)+(s+2-t-1 / t) f_{m-1} g_{m-1} \tau_{n} .
$$

Proof By [20] (see also [10, page 309]), the Riley polynomial is

$$
\phi_{K}(s, t)=z_{1,1}+(1-t) z_{1,2} \text {, }
$$

where $z_{i, j}$ is the $(i, j)$-entry of $W^{n}$. From Lemma 3.6 and the recursive formula for $\tau_{k}$,

$$
\begin{aligned}
\phi_{K}(s, t) & =\left(w_{1,1} \tau_{n}-\tau_{n-1}\right)+(1-t) w_{1,2} \tau_{n} \\
& =\tau_{n+1}-(\operatorname{tr} W) \tau_{n}+\left(w_{1,1}+(1-t) w_{1,2}\right) \tau_{n} \\
& =\left(\tau_{n+1}-\tau_{n}\right)+\left(w_{1,1}+(1-t) w_{1,2}+1-\operatorname{tr} W\right) \tau_{n} .
\end{aligned}
$$

Recall that by Proposition 3.2, $\operatorname{tr} W=f_{m}^{2}+f_{m-1}^{2}-s(t+1 / t) g_{m-1}^{2}$. Thus we have $w_{1,1}+(1-t) w_{1,2}+1-\operatorname{tr} W$

$$
\begin{aligned}
& =(1-t) f_{m-1} g_{m-1}-\frac{1-t}{t} f_{m} g_{m-1}+1-f_{m-1}^{2}+\frac{s}{t} g_{m-1}^{2} \\
& =(2-t-1 / t) f_{m-1} g_{m-1}+1-f_{m-1}^{2}+s g_{m-1}^{2}
\end{aligned}
$$

from Lemma 2.3(2). Lemma 2.3(1) and (3) give

$$
\begin{aligned}
1-f_{m-1}^{2}+s g_{m-1}^{2} & =-s g_{m-1} g_{m-2}+s g_{m-1}^{2} \\
& =s g_{m-1}\left(g_{m-1}-g_{m-2}\right)=s g_{m-1} f_{m-1} .
\end{aligned}
$$

Thus we obtain $\phi_{K}(s, t)=\left(\tau_{n+1}-\tau_{n}\right)+(s+2-t-1 / t) f_{m-1} g_{m-1} \tau_{n}$.

For convenience, we introduce a variable $T=t+1 / t$. Then the Riley polynomial of $K$ is expressed as $\phi_{K}(s, T)=\left(\tau_{n+1}-\tau_{n}\right)+(s+2-T) f_{m-1} g_{m-1} \tau_{n}$.

We remark that the Riley polynomial of $K$ is also described by Morifuji and Tran [17] in a different form. 
Example 3.8 If $n=1$, then

$$
\begin{aligned}
\phi_{K}(s, T) & =\left(\tau_{2}-\tau_{1}\right)+(s+2-T) f_{m-1} g_{m-1} \tau_{1} \\
& =(\operatorname{tr} W-1)+(s+2-T) f_{m-1} g_{m-1} \\
& =s(s+2-T) g_{m-1}^{2}+1+(s+2-T) f_{m-1} g_{m-1} \\
& =(s+2-T) g_{m-1}\left(s g_{m-1}+f_{m-1}\right)+1=(s+2-T) g_{m-1} f_{m}+1 .
\end{aligned}
$$

Thus Riley's equation $\phi_{K}(s, T)=0$ has the unique solution $T=s+2+1 /\left(f_{m} g_{m-1}\right)$ for any $s>0$. Then $T>s+2>2$, because $f_{m}>0$ and $g_{m-1}>0$. Hence we have a real solution $t=\left(T+\sqrt{T^{2}-4}\right) / 2>1$. By Lemma 2.4, we also have $s+2<T<s+2+4 /\left(s g_{m-1}^{2}\right)$. Thus $s$ and $t$ satisfy Assumption 3.1.

Remark 3.9 Formally, the conclusions of Lemma 3.6 and Proposition 3.7 are true even for $n=-1$. Then $\phi_{K}(s, T)=1+(T-s-2) f_{m-1} g_{m-1}$. Hence the equation $\phi_{K}(s, T)=0$ has the unique solution $T=s+2-1 /\left(f_{m-1} g_{m-1}\right)$, which is smaller than $s+2$. The requirement $T>s+2$ plays a crucial role in Proposition 5.2 and Section 6 . This is the reason why the case $n=-1$ should be excluded.

\section{Solutions of Riley's equation}

In this section, we examine whether Riley's equation $\phi_{K}(s, T)=0$ has a pair of real solutions $(s, T)$ satisfying Assumption 3.1. In fact, we can choose $T$ satisfying $s+2+c /\left(s g_{m-1}^{2}\right)<T<s+2+d /\left(s g_{m-1}^{2}\right)$, where $c$ and $d$ are constants depending only $n$, for any $s>0$, unless $n= \pm 1$. The case where $n=1$ is covered in Example 3.8. Let $k$ be a positive integer. For $z=e^{i \theta}(0 \leq \theta \leq \pi)$, set $\mathcal{T}_{k}(z)=z^{k-1}+z^{k-3}+$ $\cdots+z^{3-k}+z^{1-k}$. If $z \neq \pm 1$, then $\mathcal{T}_{k}(z)=\left(z^{k}-z^{-k}\right) /\left(z-z^{-1}\right)$. Define $\mathcal{T}_{0}=0$ and $\mathcal{T}_{-k}(z)=-\mathcal{T}_{k}(z)$. Since $\mathcal{T}_{k}(z)$ is symmetric for $z$ and $z^{-1}$, it can be expanded as a polynomial of $z+z^{-1}$. Furthermore, a recursive relation

$$
\mathcal{T}_{k+1}(z)-\left(z+z^{-1}\right) \mathcal{T}_{k}(z)+\mathcal{T}_{k-1}(z)=0
$$

holds. Also, $\mathcal{T}_{k}(1)=k$ and $\mathcal{T}_{k}(-1)=(-1)^{k-1} k$ for any integer $k$.

Lemma 4.1 (1) Let $k \geq 1$. Then $\mathcal{T}_{k}\left(e^{\frac{\pi}{2 k+1} i}\right)=\mathcal{T}_{k+1}\left(e^{\frac{\pi}{2 k+1} i}\right)$. This value is positive.

(2) Let $k \geq 2$. Then $\mathcal{T}_{k}\left(e^{\frac{3 \pi}{2 k+1} i}\right)=\mathcal{T}_{k+1}\left(e^{\frac{3 \pi}{2 k+1} i}\right)$. This value is negative.

Proof (1) Let $z=e^{\frac{\pi}{2 k+1} i}$. Then $z^{k} \cdot z^{k+1}=-1$. Hence

$$
\mathcal{T}_{k}(z)=\frac{z^{k}-z^{-k}}{z-z^{-1}}=\frac{-z^{-k-1}+z^{k+1}}{z-z^{-1}}=\mathcal{T}_{k+1}(z) .
$$


A direct calculation shows

$$
\mathcal{T}_{k}(z)=\frac{2 \operatorname{Im}\left(z^{k}\right) i}{2 \operatorname{Im}(z) i}=\frac{\sin \frac{k \pi}{2 k+1}}{\sin \frac{\pi}{2 k+1}}>0 .
$$

(2) Similarly, set $z=e^{\frac{3 \pi}{2 k+1} i}$. Then $z^{3 k} \cdot z^{3 k+1}=-1$. Hence we have $\mathcal{T}_{k}(z)=$ $\mathcal{T}_{k+1}(z)$ again, and

$$
\mathcal{T}_{k}(z)=\frac{\sin \frac{3 k \pi}{2 k+1}}{\sin \frac{3 \pi}{2 k+1}}<0 .
$$

Now, fix an $s>0$. We introduce a function $\Phi:\left[s+2, s+2+4 /\left(s g_{m-1}^{2}\right)\right] \rightarrow \mathbb{R}$ by

$$
\Phi(T)=\left(\mathcal{T}_{n+1}(z)-\mathcal{T}_{n}(z)\right)+(s+2-T) f_{m-1} g_{m-1} \mathcal{T}_{n}(z),
$$

where $z=\left(\tau+i \sqrt{4-\tau^{2}}\right) / 2$ with $\tau=s(s+2-T) g_{m-1}^{2}+2$. Note that $-2 \leq \tau \leq 2$. We will seek a solution $T$ for $\Phi(T)=0$ satisfying $s+2<T<s+2+4 /\left(s g_{m-1}^{2}\right)$. For a solution $T, \tau=\operatorname{tr} W$ (see the proof of Lemma 3.3) and $z$ gives an eigenvalue of $W$. Hence $\mathcal{T}_{k}(z)=\tau_{k}$, and so we have a pair of solutions $(s, T)$ for Riley's equation $\phi_{K}(s, T)=0$ satisfying Assumption 3.1.

Proposition 4.2 Suppose $n \neq \pm 1$. For any $s>0$, Riley's equation $\phi_{K}(s, T)=0$ has a solution $T$ satisfying $s+2+c /\left(s g_{m-1}^{2}\right)<T<s+2+d /\left(s g_{m-1}^{2}\right)$, where $c$ and $d$ are constants in $(0,4)$ depending only on $n$. In particular, $\phi_{K}(s, t)=0$ has a solution $t>0$ for any $s>0$ satisfying Assumption 3.1.

Proof Suppose $n>1$. By Lemma 4.1,

$$
\mathcal{T}_{n+1}\left(e^{\frac{\pi}{2 n+1} i}\right)=\mathcal{T}_{n}\left(e^{\frac{\pi}{2 n+1} i}\right), \quad \mathcal{T}_{n+1}\left(e^{\frac{3 \pi}{2 n+1} i}\right)=\mathcal{T}_{n}\left(e^{\frac{3 \pi}{2 n+1} i}\right) .
$$

Let $c=2-2 \cos \frac{\pi}{2 n+1}$ and $c^{\prime}=2-2 \cos \frac{3 \pi}{2 n+1}$. Then $c, c^{\prime} \in(0,4)$ and $c<c^{\prime}$.

If $T=s+2+c /\left(s g_{m-1}^{2}\right)\left(\right.$ resp. $\left.s+2+c^{\prime} /\left(s g_{m-1}^{2}\right)\right)$ then $\tau=2-c$ (resp. $\left.2-c^{\prime}\right)$. Thus

$$
\begin{aligned}
& \Phi\left(s+2+\frac{c}{s g_{m-1}^{2}}\right)=-\frac{c f_{m-1}}{s g_{m-1}} \mathcal{T}_{n}\left(e^{\frac{\pi}{2 n+1} i}\right), \\
& \Phi\left(s+2+\frac{c^{\prime}}{s g_{m-1}^{2}}\right)=-\frac{c^{\prime} f_{m-1}}{s g_{m-1}} \mathcal{T}_{n}\left(e^{\frac{3 \pi}{2 n+1} i}\right) .
\end{aligned}
$$

By Lemma 4.1, these values have distinct signs. We remark that $\Phi(T)$ is a polynomial function of $T$, so it is continuous. Thus if $n>1$, we have a solution $T$ for $\Phi(T)=0$, satisfying $s+2+c /\left(s g_{m-1}^{2}\right)<T<s+2+c^{\prime} /\left(s g_{m-1}^{2}\right)$, from the intermediate 
value theorem. Since $T>2, t+1 / t=T$ has a real solution for $t$. If we choose $t=\left(T+\sqrt{T^{2}-4}\right) / 2$, then $t>1$.

Suppose $n<-1$. Set $l=|n|$. If $l>2$, then set $d=2-2 \cos \frac{\pi}{2 l-1}$ and $d^{\prime}=$ $2-2 \cos \frac{3 \pi}{2 l-1}$. Then $d, d^{\prime} \in(0,4)$ and $d<d^{\prime}$. As before,

$$
\mathcal{T}_{l-1}\left(e^{\frac{\pi}{2 l-1} i}\right)=\mathcal{T}_{l}\left(e^{\frac{\pi}{2 l-1} i}\right), \quad \mathcal{T}_{l-1}\left(e^{\frac{3 \pi}{2 l-1} i}\right)=\mathcal{T}_{l}\left(e^{\frac{3 \pi}{2 l-1} i}\right),
$$

by Lemma 4.1. Thus

$$
\begin{aligned}
& \Phi\left(s+2+\frac{d}{s g_{m-1}^{2}}\right)=\frac{d f_{m-1}}{s g_{m-1}^{2}} \mathcal{T}_{l}\left(e^{\frac{\pi}{2 l-1} i}\right), \\
& \Phi\left(s+2+\frac{d^{\prime}}{s g_{m-1}^{2}}\right)=\frac{d^{\prime} f_{m-1}}{s g_{m-1}^{2}} \mathcal{T}_{l}\left(e^{\frac{3 \pi}{2 l-1} i}\right) .
\end{aligned}
$$

Since these values have distinct signs, we have a solution $T$ with $s+2+d /\left(s g_{m-1}^{2}\right)<$ $T<s+2+d^{\prime} /\left(s g_{m-1}^{2}\right)$, if $l>2$, as before.

When $l=2$, we have

$$
\Phi\left(s+2+\frac{1}{s g_{m-1}^{2}}\right)=\frac{f_{m-1}}{s g_{m-1}}>0, \quad \Phi\left(s+2+\frac{2}{s g_{m-1}^{2}}\right)=-1 .
$$

Therefore there exists a solution $T$ with $s+2+1 /\left(s g_{m-1}^{2}\right)<T<s+2+2 /\left(s g_{m-1}^{2}\right)$.

By Proposition 4.2, we have a representation $\rho: G \rightarrow \mathrm{SL}_{2}(\mathbb{R})$ for any $s>0$.

\section{Longitudes}

For any $s>0$, we choose $t$ so that $\phi_{K}(s, t)=0$. Let $\rho_{s}: G \rightarrow \mathrm{SL}_{2}(\mathbb{R})$ be the representation defined by the correspondence

$$
\rho_{s}(x)=\left(\begin{array}{cc}
\sqrt{t} & 0 \\
0 & \frac{1}{\sqrt{t}}
\end{array}\right), \quad \rho_{s}(y)=\left(\begin{array}{cc}
\frac{t-s-1}{\sqrt{t}-\frac{1}{\sqrt{t}}} & \frac{s}{\left(\sqrt{t}-\frac{1}{\sqrt{t}}\right)^{2}}-1 \\
-s & \frac{s+1-\frac{1}{t}}{\sqrt{t}-\frac{1}{\sqrt{t}}}
\end{array}\right) .
$$

For $Q=\left(\begin{array}{cc}t-1 & 1 \\ 0 & \sqrt{t}-\frac{1}{\sqrt{t}}\end{array}\right)$,

$$
Q^{-1} \rho_{s}(x) Q=\left(\begin{array}{cc}
\sqrt{t} & \frac{1}{\sqrt{t}} \\
0 & \frac{1}{\sqrt{t}}
\end{array}\right), \quad Q^{-1} \rho_{s}(y) Q=\left(\begin{array}{cc}
\sqrt{t} & 0 \\
-s \sqrt{t} & \frac{1}{\sqrt{t}}
\end{array}\right) .
$$


Therefore, $\rho_{s}$ is conjugate with $\rho$ defined in the previous section. This implies that if $s$ and $t$ satisfy Riley's equation $\phi_{K}(s, t)=0$ then $\rho_{s}$ gives a representation of $G$, as well as $\rho$. Hence we need to assume $n \neq-1$. In this section, we examine the image of the longitude $\mathcal{L}$ of $G$ under $\rho_{s}$.

Throughout the section, let $U=\rho_{s}(w)$ and $u_{i, j}$ be its $(i, j)$-entry, and let $v_{i, j}$ be the entries of $U^{n}$. Also, set

$$
\sigma=\frac{s\left(\sqrt{t}-\frac{1}{\sqrt{t}}\right)^{2}}{\left(\sqrt{t}-\frac{1}{\sqrt{t}}\right)^{2}-s} .
$$

Lemma 5.1 For $w_{*}^{n}$, we have

$$
\rho_{S}\left(w_{*}^{n}\right)=\left(\begin{array}{cc}
v_{1,1} & \frac{v_{2,1}}{\sigma} \\
v_{1,2} \sigma & v_{2,2}
\end{array}\right) .
$$

Proof By a direct calculation,

$$
\begin{array}{ll}
\rho_{s}\left(x y^{-1}\right)=\left(\begin{array}{cc}
\frac{t-1+s t}{t-1} & \frac{s \sqrt{t}}{\sigma} \\
\frac{s}{\sqrt{t}} & \frac{t-1-s}{t-1}
\end{array}\right), & \rho_{s}\left(y^{-1} x\right)=\left(\begin{array}{cc}
\frac{t-1+s t}{t-1} & \frac{s}{\sqrt{t} \sigma} \\
s \sqrt{t} & \frac{t-1-s}{t-1}
\end{array}\right), \\
\rho_{s}\left(x^{-1} y\right)=\left(\begin{array}{cc}
\frac{t-1-s}{t-1} & -\frac{s}{\sqrt{t} \sigma} \\
-s \sqrt{t} & \frac{t-1+s t}{t-1}
\end{array}\right), & \rho_{s}\left(y x^{-1}\right)=\left(\begin{array}{cc}
\frac{t-1-s}{t-1} & -\frac{s \sqrt{t}}{\sigma} \\
-\frac{s}{\sqrt{t}} & \frac{t-1+s t}{t-1}
\end{array}\right) .
\end{array}
$$

Thus we see that the $(1,2)$-entry of $\rho_{s}\left(y^{-1} x\right)$ is the $(2,1)$-entry of $\rho_{s}\left(x y^{-1}\right)$ divided by $\sigma$, the $(2,1)$-entry of $\rho_{s}\left(y^{-1} x\right)$ is the $(1,2)$-entry of $\rho_{s}\left(x y^{-1}\right)$ multiplied by $\sigma$, and the others of $\rho_{s}\left(y^{-1} x\right)$ coincide with those of $\rho_{s}\left(x y^{-1}\right)$. The same relation between entries holds for $\rho_{s}\left(x^{-1} y\right)$ and $\rho_{s}\left(y x^{-1}\right)$.

In general, such a relation is preserved under the matrix multiplication;

$$
\left(\begin{array}{cc}
p & \frac{r}{\sigma} \\
q \sigma & s
\end{array}\right)\left(\begin{array}{cc}
a & \frac{c}{\sigma} \\
b \sigma & d
\end{array}\right)=\left(\begin{array}{cc}
a p+b r & \frac{c p+d r}{\sigma} \\
(a q+b s) \sigma & c q+d s
\end{array}\right)
$$

Thus we can confirm that the same relation holds for $\rho_{s}\left(w^{n}\right)$ and $\rho_{s}\left(w_{*}^{n}\right)$.

Proposition 5.2 For the longitude $\mathcal{L}$ of $G$, the matrix $\rho_{S}(\mathcal{L})$ is diagonal, and the $(1,1)$-entry of $\rho_{S}(\mathcal{L})$ is a positive real number.

Proof The first assertion follows from the facts that for a meridian $x, \rho_{S}(x)$ is diagonal but $\rho_{s}(x) \neq \pm I$ and that $x$ and $\mathcal{L}$ commute. 
Since $\mathcal{L}=w_{*}^{n} w^{n}$, Lemma 5.1 implies that

$$
\begin{aligned}
\rho_{S}(\mathcal{L})=\rho_{s}\left(w_{*}^{n}\right) \rho_{s}\left(w^{n}\right) & =\left(\begin{array}{cc}
v_{1,1} & \frac{v_{2,1}}{\sigma} \\
v_{1,2} \sigma & v_{2,2}
\end{array}\right)\left(\begin{array}{ll}
v_{1,1} & v_{1,2} \\
v_{2,1} & v_{2,2}
\end{array}\right) \\
& =\left(\begin{array}{cc}
v_{1,1}^{2}+\frac{v_{2,1}^{2}}{\sigma} & v_{1,1} v_{1,2}+\frac{v_{2,1} v_{2,2}}{\sigma} \\
v_{1,1} v_{1,2} \sigma+v_{2,1} v_{2,2} & v_{1,2}^{2} \sigma+v_{2,2}^{2}
\end{array}\right) .
\end{aligned}
$$

Since $\operatorname{det} \rho_{S}\left(w^{n}\right)=1$, at least one of $v_{1,1}$ and $v_{2,1}$ is non-zero. Hence the $(1,1)$-entry is $v_{1,1}^{2}+v_{2,1}^{2} / \sigma$, which is positive, because $s>0$ and $(\sqrt{t}-1 / \sqrt{t})^{2}-s=T-s-2>0$ by Proposition 4.2 and Example 3.8.

Remark 5.3 Since $\rho_{S}(\mathcal{L})$ is diagonal, we also obtain an equation

$$
v_{1,1} v_{1,2} \sigma+v_{2,1} v_{2,2}=0
$$

from the $(2,1)$-entry of $\rho_{s}(\mathcal{L})$. This will be used in the proof of Lemma 5.6.

For $W=\rho(w)$ in Section 3, recall that the entries are denoted $w_{i, j}$.

Lemma 5.4 For $U=\rho_{s}(w)$,

$$
\begin{array}{ll}
u_{1,1}=w_{1,1}+\frac{w_{2,1}}{t-1}, & u_{1,2}=\sqrt{t}\left(w_{1,2}-\frac{w_{1,1}}{t-1}\right)+\frac{\sqrt{t}}{t-1}\left(w_{2,2}-\frac{w_{2,1}}{t-1}\right), \\
u_{2,1}=\frac{w_{2,1}}{\sqrt{t}}, & u_{2,2}=w_{2,2}-\frac{w_{2,1}}{t-1} .
\end{array}
$$

Proof This immediately follows by calculating the product $U=Q W Q^{-1}$.

By Lemma 3.6, we have

$$
W^{n}=\left(\begin{array}{cc}
w_{1,1} \tau_{n}-\tau_{n-1} & w_{1,2} \tau_{n} \\
w_{2,1} \tau_{n} & \tau_{n+1}-w_{1,1} \tau_{n}
\end{array}\right)
$$

Lemma 5.5 For $U^{n}=\rho_{s}\left(w^{n}\right)$, we have

$$
v_{1,1}=u_{1,1} \tau_{n}-\tau_{n-1}, \quad v_{1,2}=u_{1,2} \tau_{n}, \quad v_{2,1}=u_{2,1} \tau_{n}, \quad v_{2,2}=\tau_{n+1}-u_{1,1} \tau_{n} .
$$

Proof Calculate the product $U^{n}=Q W^{n} Q^{-1}$. Then

$$
v_{1,1}=\left(w_{1,1}+w_{2,1} /(t-1)\right) \tau_{n}-\tau_{n-1}=u_{1,1} \tau_{n}-\tau_{n-1}
$$


by Lemma 5.4. For $v_{1,2}$, we have

$$
\begin{aligned}
v_{1,2}=\frac{\sqrt{t}}{(t-1)^{2}}\left((t-1) \tau_{n-1}-(t-1) w_{1,1} \tau_{n}\right. & +(t-1)^{2} w_{1,2} \tau_{n} \\
& \left.+(t-1) \tau_{n+1}-(t-1) w_{1,1} \tau_{n}-w_{2,1} \tau_{n}\right) .
\end{aligned}
$$

Recall that the Riley polynomial is $\phi_{K}(s, t)=\left(w_{1,1} \tau_{n}-\tau_{n-1}\right)+(1-t) w_{1,2} \tau_{n}$. (See the proof of Proposition 3.7.) Since $\phi_{K}(s, t)=0, \tau_{n-1}=w_{1,1} \tau_{n}+(1-t) w_{1,2} \tau_{n}$. Hence

$$
\begin{aligned}
v_{1,2} & =\frac{\sqrt{t}}{(t-1)^{2}}\left((t-1) \tau_{n+1}-\left(w_{2,1}+(t-1) w_{1,1}\right) \tau_{n}\right) \\
& =\frac{\sqrt{t}}{(t-1)^{2}}\left(\left((t-1) w_{2,2}-w_{2,1}\right) \tau_{n}-(t-1) \tau_{n-1}\right) \\
& =\frac{\sqrt{t}}{t-1}\left(\left(w_{2,2}-\frac{w_{2,1}}{t-1}\right) \tau_{n}-\tau_{n-1}\right)=\frac{\sqrt{t}}{t-1}\left(u_{2,2} \tau_{n}-\tau_{n-1}\right),
\end{aligned}
$$

by using the recursion $\tau_{n+1}-\left(w_{1,1}+w_{2,2}\right) \tau_{n}+\tau_{n-1}=0$. By Lemma 5.4,

$$
u_{2,2}=\frac{t-1}{\sqrt{t}}\left(u_{1,2}-\sqrt{t}\left(w_{1,2}-\frac{w_{1,1}}{t-1}\right)\right) .
$$

After substituting this and $\phi_{K}(s, t)=0$, we have

$$
v_{1,2}=u_{1,2} \tau_{n}+\frac{\sqrt{t}}{t-1}\left(w_{1,1} \tau_{n}-\tau_{n-1}+(1-t) w_{1,2} \tau_{n}\right)=u_{1,2} \tau_{n}
$$

It is straightforward to check $v_{2,1}$ and $v_{2,2}$. We omit them.

Let $B_{S}$ be the $(1,1)$-entry of the matrix $\rho_{S}(\mathcal{L})$. By the proof of Proposition 5.2, $B_{S}=v_{1,1}^{2}+v_{2,1}^{2} / \sigma$.

\section{Lemma 5.6 $B_{s}=-u_{2,1} /\left(u_{1,2} \sigma\right)$}

Proof As noted in Remark 5.3, $v_{1,1} v_{1,2} \sigma+v_{2,1} v_{2,2}=0$. Since $\operatorname{det} U^{n}=v_{1,1} v_{2,2}-$ $v_{1,2} v_{2,1}=1$, we have

$$
\begin{aligned}
v_{1,2} B_{S} & =v_{1,1}^{2} v_{1,2}+\frac{v_{1,2} v_{2,1}^{2}}{\sigma}=v_{1,1}^{2} v_{1,2}+\frac{v_{2,1}}{\sigma}\left(v_{1,1} v_{2,2}-1\right) \\
& =v_{1,1}^{2} v_{1,2}+\frac{v_{1,1}}{\sigma}\left(-v_{1,1} v_{1,2} \sigma\right)-\frac{v_{2,1}}{\sigma}=-\frac{v_{2,1}}{\sigma} .
\end{aligned}
$$

Assume $v_{1,2}=0$. Then $v_{2,1}=0$. By Lemmas 5.4 and 5.5, $v_{2,1}=u_{2,1} \tau_{n}=w_{2,1} \tau_{n} / \sqrt{t}$. Since $w_{2,1} \neq 0$ by Lemma 3.5, we have $\tau_{n}=0$. Recall that

$$
\phi_{K}(s, t)=\left(\tau_{n+1}-\tau_{n}\right)+(s+2-t-1 / t) f_{m-1} g_{m-1} \tau_{n}
$$


is zero. Thus $\tau_{n+1}=0$. Then the recursive formula for $\tau_{k}$ implies $\tau_{n-1}=0$. In turn, all $\tau_{k}=0$. But this is impossible, because $\tau_{1}=1$. Hence $v_{1,2} \neq 0$, so $B_{s}=-v_{2,1} /\left(v_{1,2} \sigma\right)$.

By Lemma 5.5, $v_{1,2}=u_{1,2} \tau_{n}$ and $v_{2,1}=u_{2,1} \tau_{n}$. Thus we have shown that $B_{s}=$ $-u_{2,1} /\left(u_{1,2} \sigma\right)$.

Proposition 5.7 For the longitude $\mathcal{L}$, the $(1,1)$-entry $B_{S}$ of $\rho_{S}(\mathcal{L})$ is given as

$$
B_{s}=\frac{-f_{m}+t f_{m-1}}{-f_{m-1}+t f_{m}}
$$

Proof From Lemma 5.6, $B_{s}=-u_{2,1} /\left(u_{1,2} \sigma\right)$. Then Lemma 5.4 and Proposition 3.2 give

$$
\begin{aligned}
& u_{1,2}=\frac{\sqrt{t}}{t-1}\left(f_{m-1}^{2}-f_{m}^{2}+\left(s t-\frac{s}{t}\right) g_{m-1}^{2}\right)-\frac{(t-1)^{2}+s t}{\sqrt{t}(t-1)^{2}} g_{m-1}\left(f_{m}-t f_{m-1}\right), \\
& u_{2,1}=\frac{s}{\sqrt{t}} g_{m-1}\left(f_{m}-t f_{m-1}\right) .
\end{aligned}
$$

For the first term of $u_{1,2}$, Lemma 2.3 implies

$$
\begin{aligned}
f_{m-1}^{2}-f_{m}^{2}+\left(s t-\frac{s}{t}\right) g_{m-1}^{2} & =\left(f_{m-1}-f_{m}\right)\left(f_{m-1}+f_{m}\right)+\left(t-\frac{1}{t}\right) s g_{m-1}^{2} \\
& =-s g_{m-1}\left(f_{m-1}+f_{m}\right)+\left(t-\frac{1}{t}\right) s g_{m-1}^{2} \\
& =s g_{m-1}\left(-f_{m-1}-f_{m}+\left(t-\frac{1}{t}\right) g_{m-1}\right) \\
& =s g_{m-1}\left(-f_{m-1}-f_{m}+\left(t-\frac{1}{t}\right) \frac{f_{m}-f_{m-1}}{s}\right) \\
& =s g_{m-1}\left(\frac{t^{2}-1-s t}{s t} f_{m}-\frac{t^{2}-1+s t}{s t} f_{m-1}\right) .
\end{aligned}
$$

Thus, dividing $u_{1,2}$ by $s g_{m-1} / \sqrt{t}$ gives

$$
\frac{t}{t-1}\left(\frac{t^{2}-1-s t}{s t} f_{m}-\frac{t^{2}-1+s t}{s t} f_{m-1}\right)-\frac{(t-1)^{2}+s t}{s(t-1)^{2}}\left(f_{m}-t f_{m-1}\right) .
$$

The coefficient of $f_{m}$ is $t / \sigma$, and that of $f_{m-1}$ is $-1 / \sigma$. Hence we have

$$
B_{s}=-\frac{u_{2,1}}{u_{1,2} \sigma}=\frac{-f_{m}+t f_{m-1}}{-f_{m-1}+t f_{m}} .
$$




\section{Limits}

Let $r=p / q$ be a rational number, and let $K(r)$ denote the resulting manifold by $r$-surgery on $K$. In other words, $K(r)$ is obtained by attaching a solid torus $V$ to the knot exterior $E(K)$ along their boundaries so that a loop of slope $r$ bounds a meridian disk of $V$.

Our representation $\rho_{s}: G \rightarrow \mathrm{SL}_{2}(\mathbb{R})$ induces a homomorphism $\pi_{1}(K(r)) \rightarrow \mathrm{SL}_{2}(\mathbb{R})$ if and only if $\rho_{s}(x)^{p} \rho_{s}(\mathcal{L})^{q}=I$. Since both of $\rho_{s}(x)$ and $\rho_{s}(\mathcal{L})$ are diagonal (see (6) and Proposition 5.2), this is equivalent to the single equation

$$
A_{s}^{p} B_{s}^{q}=1,
$$

where $A_{S}$ and $B_{S}$ are the $(1,1)$-entries of $\rho_{S}(x)$ and $\rho_{S}(\mathcal{L})$, respectively. We remark that $A_{s}=\sqrt{t}(>1)$ is a positive real number, as is $B_{s}$ by Proposition 5.2. Hence the equation (8) is furthermore equivalent to the equation

$$
-\frac{\log B_{S}}{\log A_{s}}=\frac{p}{q} \text {. }
$$

Let $g:(0, \infty) \rightarrow \mathbb{R}$ be a function defined by

$$
g(s)=-\frac{\log B_{s}}{\log A_{s}} .
$$

We will examine the image of $g$.

Lemma 6.1 (1) $\lim _{s \rightarrow+0} t= \begin{cases}\infty & \text { if } n \neq \pm 1 \\ \frac{2 m+1+\sqrt{4 m+1}}{2 m} & \text { if } n=1\end{cases}$

(2) $\lim _{s \rightarrow \infty} t=\infty$

(3) $\lim _{s \rightarrow \infty}(t-s)=2$

(4) $\lim _{s \rightarrow \infty} \frac{t}{s}=1$

Proof (1) If $n=1$, then $T=s+2+1 /\left(f_{m} g_{m-1}\right)$ is the unique solution for $\phi_{K}(s, T)=0$ (see Example 3.8). From Lemma 2.2, we have $\lim _{s \rightarrow+0} f_{m}=1$ and $\lim _{s \rightarrow+0} g_{m-1}=m$. Hence $\lim _{s \rightarrow+0} T=2+1 / m$. Since $t=\left(T+\sqrt{T^{2}-4}\right) / 2$, $\lim _{s \rightarrow+0} t=(2 m+1+\sqrt{4 m+1}) /(2 m)$.

Assume $n \neq \pm 1$. From Proposition 4.2, we have $s+2+c /\left(s g_{m-1}^{2}\right)<T$, where $c$ is a positive constant. Hence $\lim _{s \rightarrow+0} T=\lim _{s \rightarrow+0} t=\infty$.

(2) As $T>s+2, \lim _{s \rightarrow \infty} T=\lim _{s \rightarrow \infty} t=\infty$.

(3) Since $s+2<t+1 / t<s+2+4 /\left(s g_{m-1}^{2}\right)$, (2) implies $\lim _{s \rightarrow \infty}(t-s)=2$. 
(4) From $s+2<T<s+2+4 /\left(s g_{m-1}^{2}\right)$ again, we have $\lim _{s \rightarrow \infty} T / s=1$, which implies $\lim _{s \rightarrow \infty} t / s=1$.

Let $F_{k}=t^{k-1}\left(-f_{k}+t f_{k-1}\right)$ for any integer $k>0$.

Lemma 6.2 If $0<k \leq m$, then $\lim _{s \rightarrow \infty} F_{k}=1$.

Proof First, $F_{1}=-f_{1}+t f_{0}=t-s-1$. Hence $\lim _{s \rightarrow \infty} F_{1}=1$ by Lemma 6.1(3).

Let $n \neq \pm 1$. By Proposition 4.2, we have

$$
s+2+\frac{c}{s g_{m-1}^{2}}<t+\frac{1}{t}<s+2+\frac{d}{s g_{m-1}^{2}},
$$

where $c$ and $d$ are constants depending on only $n$. Multiplying by $t^{k-1} f_{k-1}$ in (10) gives

$(s+2) f_{k-1} t^{k-1}+\frac{c t^{k-1} f_{k-1}}{s g_{m-1}^{2}}<t^{k} f_{k-1}+t^{k-2} f_{k-1}<(s+2) f_{k-1} t^{k-1}+\frac{d t^{k-1} f_{k-1}}{s g_{m-1}^{2}}$.

Since $(s+2) f_{k-1}=f_{k}+f_{k-2}$ by the recursion,

$$
\begin{aligned}
\left(t^{k-1} f_{k-2}-t^{k-2} f_{k-1}\right)+\frac{c t^{k-1} f_{k-1}}{s g_{m-1}^{2}} & <t^{k} f_{k-1}-t^{k-1} f_{k} \\
& <\left(t^{k-1} f_{k-2}-t^{k-2} f_{k-1}\right)+\frac{d t^{k-1} f_{k-1}}{s g_{m-1}^{2}} .
\end{aligned}
$$

Then we have

$$
F_{k-1}+\frac{c t^{k-1} f_{k-1}}{s g_{m-1}^{2}}<F_{k}<F_{k-1}+\frac{d t^{k-1} f_{k-1}}{s g_{m-1}^{2}} .
$$

The degree of $f_{k-1}$ is $k-1$, but that of $g_{m-1}$ is $m-1$. Hence Lemma 6.1(4) implies

$$
\lim _{s \rightarrow \infty} \frac{c t^{k-1} f_{k-1}}{s g_{m-1}^{2}}=\lim _{s \rightarrow \infty} \frac{d t^{k-1} f_{k-1}}{s g_{m-1}^{2}}=0
$$

as long as $k \leq m$. Thus $\lim _{s \rightarrow \infty} F_{k}=1$ will follow from $\lim _{s \rightarrow \infty} F_{k-1}=1$.

Suppose $n=1$. Riley's equation has the unique solution

$$
t+\frac{1}{t}=s+2+\frac{1}{f_{m} g_{m-1}}
$$

(see Example 3.8). 
Multiplying by $t^{k-1} f_{k-1}$ in (12) gives

$$
t^{k} f_{k-1}+t^{k-2} f_{k-1}=(s+2) f_{k-1} t^{k-1}+\frac{t^{k-1} f_{k-1}}{f_{m} g_{m-1}} .
$$

Since $(s+2) f_{k-1}=f_{k}+f_{k-2}$,

$$
t^{k} f_{k-1}-t^{k-1} f_{k}=t^{k-1} f_{k-2}-t^{k-2} f_{k-1}+\frac{t^{k-1} f_{k-1}}{f_{m} g_{m-1}} .
$$

That is, $F_{k}=F_{k-1}+t^{k-1} f_{k-1} /\left(f_{m} g_{m-1}\right)$. Hence the fact that $\lim _{s \rightarrow \infty} F_{k-1}=1$ and $\lim _{s \rightarrow \infty} t^{k-1} f_{k-1} /\left(f_{m} g_{m-1}\right)=0$ imply $\lim _{s \rightarrow \infty} F_{k}=1$.

Lemma 6.3 (1) $\lim _{s \rightarrow+0} B_{s}=1$

(2) $\lim _{s \rightarrow \infty} B_{s} t^{2 m}=1$

Proof (1) By Proposition 5.7,

$$
B_{s}=\frac{-f_{m}+t f_{m-1}}{-f_{m-1}+t f_{m}}
$$

By Lemma 2.2, $\lim _{s \rightarrow+0} f_{m}=\lim _{s \rightarrow+0} f_{m-1}=1$. Thus Lemma 6.1(1) implies $\lim _{s \rightarrow+0} B_{s}=1$.

(2) We decompose $B_{S} t^{2 m}$ as

$$
B_{s} t^{2 m}=t^{m-1}\left(-f_{m}+t f_{m-1}\right) \cdot \frac{t^{m+1}}{-f_{m-1}+t f_{m}}=F_{m} \cdot \frac{t^{m+1}}{-f_{m-1}+t f_{m}} .
$$

Since the degree of $f_{k}$ is $k$ and $f_{m}$ is monic,

$$
\lim _{s \rightarrow \infty} \frac{t^{m+1}}{-f_{m-1}+t f_{m}}=1 .
$$

Then we have $\lim _{s \rightarrow \infty} B_{s} t^{2 m}=1$ by combined with Lemma 6.2.

Proposition 6.4 The image of $g$ contains an open interval $(0,4 m)$.

Proof By Lemma 6.3(1), $\lim _{s \rightarrow+0} \log B_{S}=0$. Hence

$$
\lim _{s \rightarrow+0} g(s)=-\lim _{s \rightarrow+0} \frac{\log B_{s}}{\log A_{s}}=-\lim _{s \rightarrow+0} \frac{\log B_{s}}{\log \sqrt{t}}=0 .
$$

Also, we have $\lim _{s \rightarrow \infty}\left(\log B_{s}+2 m \log t\right)=0$ by Lemma 6.3(2). Thus

$$
\lim _{s \rightarrow \infty} g(s)=-\lim _{s \rightarrow \infty} \frac{\log B_{s}}{\log A_{s}}=-\lim _{s \rightarrow \infty} \frac{2 \log B_{s}}{\log t}=4 m .
$$

Hence the image of $g$ contains an interval $(0,4 m)$. 


\section{Universal covering group}

We briefly review the description of the universal covering group of $\mathrm{SL}_{2}(\mathbb{R})$. See Bargmann [1] and Khoi [15].

Let

$$
\mathrm{SU}(1,1)=\left\{\left.\left(\begin{array}{cc}
\alpha & \beta \\
\bar{\beta} & \bar{\alpha}
\end{array}\right)|| \alpha\right|^{2}-|\beta|^{2}=1\right\}
$$

be the special unitary group over $\mathbb{C}$ of signature $(1,1)$. It is well known that $\mathrm{SU}(1,1)$ is conjugate to $\mathrm{SL}_{2}(\mathbb{R})$ in $\mathrm{GL}_{2}(\mathbb{C})$. The correspondence is given by $\psi: \mathrm{SL}_{2}(\mathbb{R}) \rightarrow$ SU(1,1), sending $A \mapsto J A J^{-1}$, where

$$
J=\left(\begin{array}{cc}
1 & -i \\
1 & i
\end{array}\right)
$$

Thus

$$
\psi:\left(\begin{array}{ll}
a & b \\
c & d
\end{array}\right) \mapsto\left(\begin{array}{ll}
\frac{a+d+(b-c) i}{2} & \frac{a-d-(b+c) i}{2} \\
\frac{a-d+(b+c) i}{2} & \frac{a+d-(b-c) i}{2}
\end{array}\right) .
$$

There is a parametrization of $\operatorname{SU}(1,1)$ by $(\gamma, \omega)$, where $\gamma=\beta / \alpha$ and $\omega=\arg \alpha$ are defined $\bmod 2 \pi$. Thus $\operatorname{SU}(1,1)=\{(\gamma, \omega)|| \gamma \mid<1,-\pi \leq \omega<\pi\}$. Topologically, $\mathrm{SU}(1,1)$ is an open solid torus $\Delta \times S^{1}$, where $\Delta=\{\gamma \in \mathbb{C}|| \gamma \mid<1\}$. The group operation is given by $(\gamma, \omega)\left(\gamma^{\prime}, \omega^{\prime}\right)=\left(\gamma^{\prime \prime}, \omega^{\prime \prime}\right)$, where

$$
\begin{aligned}
\gamma^{\prime \prime} & =\frac{\gamma^{\prime}+\gamma e^{-2 i \omega^{\prime}}}{1+\gamma \overline{\gamma^{\prime}} e^{-2 i \omega^{\prime}}}, \\
\omega^{\prime \prime} & =\omega+\omega^{\prime}+\frac{1}{2 i} \log \frac{1+\gamma \overline{\gamma^{\prime}} e^{-2 i \omega^{\prime}}}{1+\bar{\gamma} \gamma^{\prime} e^{2 i \omega^{\prime}}} .
\end{aligned}
$$

These equations come from the matrix operation. Here, the logarithm function is defined by its principal value and $\omega^{\prime \prime}$ is defined $\bmod 2 \pi$. The identity element is $(0,0)$, and the correspondence between $\left(\frac{\alpha}{\beta} \frac{\beta}{\alpha}\right)$ and $(\gamma, \omega)$ gives an isomorphism.

Now, the universal covering group $\widetilde{\mathrm{SL}_{2}(\mathbb{R})}$ of $\mathrm{SU}(1,1)$ can be described as

$$
\widetilde{\mathrm{SL}_{2}(\mathbb{R})}=\{(\gamma, \omega)|| \gamma \mid<1,-\infty<\omega<\infty\} \text {. }
$$

Thus $\widetilde{\mathrm{SL}_{2}(\mathbb{R})}$ is homeomorphic to $\Delta \times \mathbb{R}$. The group operation is given by (13) and (14) again, but $\omega^{\prime \prime}$ is not given mod $2 \pi$ anymore.

Let $\chi: \widetilde{\mathrm{SL}_{2}(\mathbb{R})} \rightarrow \mathrm{SL}_{2}(\mathbb{R})$ be the covering projection. Then ker $\chi=\{(0,2 j \pi) \mid j \in \mathbb{Z}\}$.

Lemma 7.1 The subset $(-1,1) \times\{0\}$ of $\widetilde{\mathrm{SL}_{2}(\mathbb{R})}$ forms a subgroup, where $(-1,1) \subset \Delta$. 
Proof From (13) and (14), it is straightforward to see that $(-1,1) \times\{0\}$ is closed under the group operation. For $(\gamma, 0) \in(-1,1) \times\{0\}$, its inverse is $(-\gamma, 0)$.

For the representation $\rho_{s}: G \rightarrow \mathrm{SL}_{2}(\mathbb{R})$ defined by (6),

$$
\psi\left(\rho_{s}(x)\right)=\frac{1}{2 \sqrt{t}}\left(\begin{array}{ll}
t+1 & t-1 \\
t-1 & t+1
\end{array}\right) \in \mathrm{SU}(1,1) .
$$

Thus $\psi\left(\rho_{s}(x)\right)$ corresponds to $\left(\gamma_{x}, 0\right)$, where $\gamma_{x}=(t-1) /(t+1)$. Since $t>1$, $\gamma_{x} \in(-1,1)$.

Also, for the longitude $\mathcal{L}$, by Proposition 5.2,

$$
\psi\left(\rho_{s}(\mathcal{L})\right)=\frac{1}{2}\left(\begin{array}{ll}
B_{s}+\frac{1}{B_{s}} & B_{s}-\frac{1}{B_{s}} \\
B_{s}-\frac{1}{B_{s}} & B_{s}+\frac{1}{B_{s}}
\end{array}\right), \quad B_{s}>0 .
$$

Thus $\psi\left(\rho_{s}(\mathcal{L})\right)$ corresponds to $\left(\gamma_{\mathcal{L}}, 0\right)$, where $\gamma_{\mathcal{L}}=\left(B_{s}^{2}-1\right) /\left(B_{s}^{2}+1\right)$. Clearly, $\gamma_{\mathcal{L}} \in(-1,1)$.

\section{Proof of Theorem 1.1}

Since the knot exterior $E(K)$ of $K$ satisfies $H^{2}(E(K) ; \mathbb{Z})=0$, any $\rho_{s}: G \rightarrow \mathrm{SL}_{2}(\mathbb{R})$ lifts to a representation $\tilde{\rho}_{s}: G \rightarrow \widetilde{\mathrm{SL}_{2}(\mathbb{R})}$ (Ghys [12]). Moreover, any two lifts $\tilde{\rho}_{s}$ and $\tilde{\rho}_{s}^{\prime}$ are related as

$$
\tilde{\rho}_{s}^{\prime}(g)=h(g) \tilde{\rho}_{s}(g)
$$

for some homomorphism $h: G \rightarrow \operatorname{ker} \chi \subset \widetilde{\operatorname{SL}_{2}(\mathbb{R})}$. Since ker $\chi=\{(0,2 j \pi) \mid j \in \mathbb{Z}\}$ is isomorphic to $\mathbb{Z}$, the homomorphism $h$ factors through $H_{1}(E(K))$, so it is determined only by the value $h(x)$ of a meridian $x$ (see [15]).

The following result is the key in [3], which is originally claimed in [15], for the figure eight knot. Our proof is essentially that of [3], but it is much simpler, because the values of $\psi\left(\rho_{S}(x)\right)$ and $\psi\left(\rho_{S}(\mathcal{L})\right)$ are calculated explicitly in Section 7. For simplicity, we identify $\mathrm{SL}_{2}(\mathbb{R})$ with $\mathrm{SU}(1,1)$ under $\psi$, and omit the symbol $\psi$. The fact that our knot has genus one is crucial in the proof of the next lemma.

Lemma 8.1 Let $\tilde{\rho}_{s}: G \rightarrow \widetilde{\mathrm{SL}_{2}(\mathbb{R})}$ be a lift of $\rho_{s}$. Then replacing $\tilde{\rho}_{s}$ by a representation $\tilde{\rho}_{s}^{\prime}=h \cdot \tilde{\rho}_{s}$ for some $h: G \rightarrow \widetilde{\mathrm{SL}_{2}(\mathbb{R})}$, we can suppose that $\tilde{\rho}_{s}\left(\pi_{1}(\partial E(K))\right)$ is contained in the subgroup $(-1,1) \times\{0\}$ of $\widetilde{\mathrm{SL}_{2}(\mathbb{R})}$. 
Proof Since $\chi\left(\tilde{\rho}_{s}(\mathcal{L})\right)=\left(\gamma_{\mathcal{L}}, 0\right), \tilde{\rho}_{s}(\mathcal{L})=\left(\gamma_{\mathcal{L}}, 2 j \pi\right)$ for some $j$. On the other hand, $\mathcal{L}$ is a commutator, because our knot has genus one. Therefore the inequality (5.5) of Wood [24] implies $-3 \pi / 2<2 j \pi<3 \pi / 2$. Thus we have $\tilde{\rho}_{S}(\mathcal{L})=\left(\gamma_{\mathcal{L}}, 0\right)$.

Similarly, $\tilde{\rho}_{s}(x)=\left(\gamma_{x}, 2 l \pi\right)$ for some $l$. Let us choose $h: G \rightarrow \widetilde{\mathrm{SL}_{2}(\mathbb{R})}$ so that $h(x)=(0,-2 l \pi)$. Set $\tilde{\rho}_{s}^{\prime}=h \cdot \tilde{\rho}_{s}$. Then a direct calculation shows that $\tilde{\rho}_{s}^{\prime}(x)=\left(\gamma_{x}, 0\right)$ and $\tilde{\rho}_{s}^{\prime}(\mathcal{L})=\left(\gamma_{\mathcal{L}}, 0\right)$. Since $x$ and $\mathcal{L}$ generate the peripheral subgroup $\pi_{1}(\partial E(K))$, the conclusion follows from these.

Proof of Theorem 1.1 Suppose $n \neq-1$. Let $r=p / q \in(0,4 m)$. By Proposition 6.4, we can find $s$ so that $g(s)=r$. Choose a lift $\tilde{\rho}_{s}$ of $\rho_{s}$ so that $\tilde{\rho}_{s}\left(\pi_{1}(\partial E(K))\right) \subset$ $(-1,1) \times\{0\}\left(\right.$ Lemma 8.1). Then $\rho_{s}\left(x^{p} \mathcal{L}^{q}\right)=I$, so $\chi\left(\widetilde{\rho}_{s}\left(x^{p} \mathcal{L}^{q}\right)\right)=I$. This means that $\tilde{\rho}_{s}\left(x^{p} \mathcal{L}^{q}\right)$ lies in ker $\chi=\{(0,2 j \pi) \mid j \in \mathbb{Z}\}$. Hence $\widetilde{\rho}_{s}\left(x^{p} \mathcal{L}^{q}\right)=(0,0)$. Then $\tilde{\rho}_{s}$ can induce a homomorphism

$$
\pi_{1}(K(r)) \rightarrow \widetilde{\mathrm{SL}_{2}(\mathbb{R})}
$$

with non-abelian image. Recall that $\widetilde{\mathrm{SL}_{2}(\mathbb{R})}$ is left-orderable (Bergman [2]) and any (non-trivial) subgroup of a left-orderable group is left-orderable. Since $K(r)$ is irreducible (Hatcher and Thurston [13]), $\pi_{1}(K(r))$ is left-orderable by Boyer, Rolfsen and Wiest [4, Theorem 1.1]. For $r=0, K(0)$ is irreducible (Gabai [11]) and has positive Betti number. Hence $\pi_{1}(K(0))$ is left-orderable by [4, Corollary 3.4]. Thus we have shown that any slope in $[0,4 m)$ is left-orderable for $K=K(m, n)$.

Suppose $n>0$. If we apply the above argument for $K(n, m)$, then any slope in $[0,4 n)$ is shown to be left-orderable. Since $K(n, m)$ is equivalent to the mirror image of $K(m, n)$, any slope in $(-4 n, 0]$ is left-orderable for $K(m, n)$. Thus we can conclude that $(-4 n, 4 m)$ consists of left-orderable slopes for $K=K(m, n)$ with $n>0$.

Suppose $m>1$ and $n<-1$. Since $K(m, n)$ is equivalent to $K(-n,-m)$, the argument in the first paragraph shows that any slope in $[0,-4 n)$ is left-orderable. In this case, we obtain $[0, \max \{4 m,-4 n\})$ consisting of left-orderable slopes.

Finally, consider the remaining cases. They are $K(1, n)$ with $n<-1$ and $K(m,-1)$ with $m>1$. Since $K(m,-1)$ is isotopic to $K(1,-m)$, two cases coincide. We obtain $[0,4)$ consisting of left-orderable slopes by the argument in the first paragraph. Furthermore, since these knots are twist knots, the slope 4 is also left-orderable by [22].

Proof of Corollary 1.2 If $n>0$, Theorem 1.1 gives the interval $(-4 n, 4)$. The slope 4 is left-orderable as in the proof of Theorem $1.1[6 ; 22]$. The claim for the case where $n<-1$ is just a repetition of Theorem 1.1. 


\section{References}

[1] V Bargmann, Irreducible unitary representations of the Lorentz group, Ann. of Math. 48 (1947) 568-640 MR0021942

[2] G M Bergman, Right orderable groups that are not locally indicable, Pacific J. Math. 147 (1991) 243-248 MR1084707

[3] S Boyer, C M Gordon, L Watson, On L-spaces and left-orderable fundamental groups, Math. Ann. 356 (2013) 1213-1245 MR3072799

[4] S Boyer, D Rolfsen, B Wiest, Orderable 3-manifold groups, Ann. Inst. Fourier (Grenoble) 55 (2005) 243-288 MR2141698

[5] G Burde, H Zieschang, Knots, 2nd edition, de Gruyter Studies in Mathematics 5, de Gruyter, Berlin (2003) MR1959408

[6] A Clay, T Lidman, L Watson, Graph manifolds, left-orderability and amalgamation, Algebr. Geom. Topol. 13 (2013) 2347-2368 MR3073920

[7] A Clay, M Teragaito, Left-orderability and exceptional Dehn surgery on two-bridge knots, from: "Geometry and topology down under", (C D Hodgson, W H Jaco, M G Scharlemann, S Tillmann, editors), Contemporary Mathematics 597, Amer. Math. Soc. (2013) 225-233

[8] A Clay, L Watson, On cabled knots, Dehn surgery, and left-orderable fundamental groups, Math. Res. Lett. 18 (2011) 1085-1095 MR2915469

[9] M K Da̧bkowski, J H Przytycki, A A Togha, Non-left-orderable 3-manifold groups, Canad. Math. Bull. 48 (2005) 32-40 MR2118761

[10] J Dubois, V Huynh, Y Yamaguchi, Non-abelian Reidemeister torsion for twist knots, J. Knot Theory Ramifications 18 (2009) 303-341 MR2514847

[11] D Gabai, Foliations and the topology of 3-manifolds, III, J. Differential Geom. 26 (1987) 479-536 MR910018

[12] É Ghys, Groups acting on the circle, Enseign. Math. 47 (2001) 329-407 MR1876932

[13] A Hatcher, W Thurston, Incompressible surfaces in 2-bridge knot complements, Invent. Math. 79 (1985) 225-246 MR778125

[14] J Hoste, P D Shanahan, A formula for the A-polynomial of twist knots, J. Knot Theory Ramifications 13 (2004) 193-209 MR2047468

[15] V T Khoi, A cut-and-paste method for computing the Seifert volumes, Math. Ann. 326 (2003) 759-801 MR2003451

[16] T Li, R Roberts, Taut foliations in knot complements (2013) arXiv:1211.3066v2

[17] T Morifuji, A T Tran, Twisted Alexander polynomials of 2-bridge knots for parabolic representations arXiv:1301.1101 
[18] P Ozsváth, Z Szabó, On knot Floer homology and lens space surgeries, Topology 44 (2005) 1281-1300 MR2168576

[19] T Peters, On L-spaces and non left-orderable 3-manifold groups arXiv: 0903.4495

[20] R Riley, Nonabelian representations of 2-bridge knot groups, Quart. J. Math. Oxford Ser. 35 (1984) 191-208 MR745421

[21] D Rolfsen, Knots and links, Mathematics Lecture Series 7, Publish or Perish, Berkeley, CA (1976) MR0515288

[22] M Teragaito, Left-orderability and exceptional Dehn surgery on twist knots, Canad. Math. Bull. 56 (2013) 850-859 MR3121694

[23] A T Tran, On left-orderable fundamental groups and Dehn surgeries on double twist knots arXiv:1301.2637

[24] J W Wood, Bundles with totally disconnected structure group, Comment. Math. Helv. 46 (1971) 257-273 MR0293655

Graduate School of Education, Hiroshima University 1-1-1 Kagamiyama, Higashi-hiroshima 739-8524, Japan

Department of Mathematics and Mathematics Education, Hiroshima University 1-1-1 Kagamiyama, Higashi-hiroshima 739-8524, Japan m122846@hiroshima-u.ac.jp, teragai@hiroshima-u.ac.jp

Received: 26 December 2012 Revised: 14 November 2013 